\title{
Consensus of Switched Multiagent Systems under Relative State Constraints
}

\author{
Qingling Wang ${ }^{1,2}$ \\ ${ }^{1}$ School of Automation, Southeast University, Nanjing, China \\ ${ }^{2}$ Key Laboratory of Measurement and Control of Complex Systems of Engineering, Ministry of Education, Nanjing, China
}

Correspondence should be addressed to Qingling Wang; csuwql@gmail.com

Received 31 May 2017; Accepted 7 November 2017; Published 20 November 2017

Academic Editor: Michele Scarpiniti

Copyright (c) 2017 Qingling Wang. This is an open access article distributed under the Creative Commons Attribution License, which permits unrestricted use, distribution, and reproduction in any medium, provided the original work is properly cited.

The consensus problem is presented for the switched multiagent system (MAS), where the MAS is switched between continuousand discrete-time systems with relative state constraints. With some standard assumptions, we obtain the fact that the switched MAS with relative state constraints can achieve consensus under both fixed undirected graphs and switching undirected graphs. Furthermore, based on the absolute average value of initial states, we propose sufficient conditions for consensus of the switched MAS. The challenge of this study is that relative state constraints are considered, which will make the consensus problem much more complex. One of the main contributions is that, for the switched MAS with relative state constraints, we explore the solvability of the consensus problem. Finally, we present two simulation examples to show the effectiveness of the results.

\section{Introduction}

Inspired by the works of $[1,2]$, the attention to the consensus problem of the MAS has grown in the research community. The definition of consensus implies that all the agents shall converge to a common value using a distributed interaction among these agents $[3,4]$. The practical applications of consensus are diverse and can be found in many fields, such as biology, physics, control systems, and robotics [5-11]. The consensus problem was first discussed for first order [12$15]$ and then generalized to second order [16, 17], general linear dynamics $[18,19]$, and nonlinear dynamics $[20,21]$ in communication networks and sensor networks.

In applications, many dynamical systems interact with the environment or have physical limitations. As such, they are often affected by saturation constraints that can disrupt consensus. Therefore, the consensus problem of the MAS subject to constraints is practically important. For example, the consensus subject to input constraints was considered in [22-27], while, for the consensus with relative state constraints, only a few results were found $[28,29]$. It has been known that the consensus subject to state constraints cannot be achieved due to saturations, such as the consensus with state/output saturation in [30].
It is observed that the above-mentioned MAS is focused on a single type of systems, that is, single continuous-time MAS or single discrete-time MAS. In fact, a large number of systems can be found where there exist both continuousand discrete-time MASs simultaneously. More recently, [31] studied the stability problem of single switched systems, while the consensus of a switched MAS was presented in $[32,33]$. Furthermore, there are other references concerning the switched MAS. For the switched MAS, [34] studied the finite-time control problem, while the containment consensus problem was presented in [35]. However, saturation constraints are not considered in the stability or consensus analysis of switched systems [31, 32].

Motivated by the work discussed above, we study the consensus of the switched MAS under the fixed undirected graph and the switching undirected graph. Firstly, by using some standard assumptions, the consensus shall be reached for the switched MAS under fixed undirected graph with the assumption that the absolute value of initial states is bounded. We next extend the consensus results to switched undirected graphs with a similar condition of initial states of agents. The main contributions are stated as follows: Firstly, compared with existing results [32], the challenge of this study is 
that state constraints are considered in the switched MAS, which increases the complexity. Secondly, the solvability of consensus problem is explored for the switched MAS with state constraints.

In what follows, we formulate the consensus problem for the switched MAS in Section 2, while the main results are illustrated in Section 3. Section 4 provides two examples to show the effectiveness, and Section 5 concludes the results of this paper.

Notation. The $\operatorname{sign}(y)$ equals to +1 when $y \geq 0$ and -1 when $y<0$. Let $\operatorname{diag}\left\{\omega_{1}, \omega_{2}, \ldots, \omega_{n}\right\}$ be a diagonal matrix, and its entries are $\omega_{i}, i=1,2, \ldots, n . P>0$ implies a positive definite matrix.

\section{Problem Formulation}

In the beginning, the graph theory will be introduced. A graph $\mathscr{G}$ can be defined as a three-tuple $(\mathscr{V}, \mathscr{E}, \mathscr{A})$, where $\mathscr{V}$ is the node set as $\mathscr{V}=\left\{v_{1}, v_{2}, \ldots, v_{N}\right\}, \mathscr{E} \subseteq \mathscr{V} \times \mathscr{V}$ is the edge set, and $\mathscr{A}=\left[a_{i j}\right] \in \mathbb{R}^{N \times N}$, where $a_{i j}$ is the underlying weighted adjacency matrix defined as $a_{i j}>0$ if $(i, j) \in \mathscr{E}$ and $a_{i j}=0$ otherwise. The set of neighbors of node $v_{i}$ is denoted by $\mathcal{N}_{i}=\left\{v_{i} \in \mathscr{V}:\left(v_{i}, v_{j}\right) \in \mathscr{E}\right\}$. The Laplacian matrix of the graph is defined as $L=\mathscr{D}-\mathscr{A}$, where $\mathscr{D}=\left[d_{i j}\right] \in \mathbb{R}^{N \times N}$ is a diagonal matrix, with $d_{i i}=\sum_{j \in \mathscr{V}} a_{i j}$. In this paper, the undirected and connected graph $\mathscr{G}$ will be considered, where its $L$ is positive semidefinite and its eigenvalues are presented by $0=\lambda_{1}<\lambda_{2} \leq \cdots \leq \lambda_{N}$.

In what follows, we consider the switched MAS, which are given as follows:

$$
\dot{x}_{i}(t)=u_{i}(t), \quad i=1,2, \ldots, N, t \in \mathbb{R}^{+},
$$

for the continuous-time systems, and

$$
x_{i}(t+1)=x_{i}(t)+u_{i}(t), \quad i=1,2, \ldots, N, t \in \mathbb{N},
$$

for the discrete-time systems, where $x_{i}(t) \in \mathbb{R}$ is the state and $u_{i}(t) \in \mathbb{R}$ in the control input. To achieve the consensus of the above switched MAS, the following control protocol from [32] has been proposed:

$$
\begin{aligned}
& u_{i}(t) \\
& = \begin{cases}\sum_{j \in \mathscr{V}} a_{i j}(t)\left(x_{j}(t)-x_{i}(t)\right), & i=1,2, \ldots, N, t \in \mathbb{R}^{+} \\
h \sum_{j \in \mathscr{V}} a_{i j}(t)\left(x_{j}(t)-x_{i}(t)\right), & i=1,2, \ldots, N, t \in \mathbb{N},\end{cases}
\end{aligned}
$$

where $h>0$ is the sampling period. However, in practice, the measurement part may have bounded nonlinearities or saturation constraints due to sensor limitations. Therefore, we will consider the consensus of the switched MAS with relative state constraints. That is, for each $i=1,2, \ldots, N$, the following control protocol will be considered:

$$
\begin{aligned}
& u_{i}(t) \\
& = \begin{cases}\sum_{j \in \mathscr{V}} a_{i j}(t)\left(\operatorname{sat}_{M}\left(x_{j}(t)\right)-\operatorname{sat}_{M}\left(x_{i}(t)\right)\right), & t \in \mathbb{R}^{+} \\
h \sum_{j \in \mathscr{V}} a_{i j}(t)\left(\operatorname{sat}_{M}\left(x_{j}(t)\right)-\operatorname{sat}_{M}\left(x_{i}(t)\right)\right), & t \in \mathbb{N} .\end{cases}
\end{aligned}
$$

Then, for each $i$, the closed-loop switched MASs with state constraints are given as

$$
\begin{aligned}
& \dot{x}_{i}(t)= \sum_{j \in \mathscr{V}} a_{i j}\left(\operatorname{sat}_{M}\left(x_{j}(t)\right)-\operatorname{sat}_{M}\left(x_{i}(t)\right)\right), \\
& t \in \mathbb{R}^{+}, \\
& x_{i}(t+1)= x_{i}(t) \\
&+h \sum_{j \in \mathscr{V}} a_{i j}\left(\operatorname{sat}_{M}\left(x_{j}(t)\right)-\operatorname{sat}_{M}\left(x_{i}(t)\right)\right), \\
& t \in \mathbb{N},
\end{aligned}
$$

where the function $\operatorname{sat}_{M}(\cdot)$ is described as a saturated characteristic given by

$$
\operatorname{sat}_{M}(x)= \begin{cases}x, & \text { if }|x| \leq M \\ M \operatorname{sign}(x), & \text { if }|x|>M\end{cases}
$$

where $M>0$ is the saturation limit. At any time instant, the activated subsystem can be chosen by a switching rule, where we consider the arbitrary switching rule in this paper. For convenience, let $i=1,2, \ldots, N$ be $i \in \mathscr{I}_{N}$.

Definition 1. Consider $N$ agents, and the network of these agents is given by a graph $\mathscr{G}$. The switched MAS with (5) and (6) is said to achieve the consensus problem, if

$$
\lim _{t \rightarrow \infty}\left\|x_{i}(t)-x_{j}(t)\right\|=0
$$

for all initial conditions $x_{i}(0) \in \mathbb{R}, i \in \mathscr{I}_{N}$.

It is worth pointing out that the switched MAS with (5) and (6) cannot achieve consensus directly. Therefore, in this paper, we will find the conditions, with which the consensus of the switched MAS with state constraints can be reached.

\section{Main Results}

This section is to derive some conditions for consensus of the switched MAS with fixed and switching undirected graphs.

In what follows, consensus of the switched MAS on fixed undirected graphs is considered; that is, $\mathscr{G}(t)=\mathscr{G}$ for any $t$. We firstly introduce a lemma, which is used in the following sections.

Lemma 2. Assume that graph $\mathscr{G}$ is connected. Consider agent (5) or (6); the average state value $(1 / N) \sum_{i=1}^{N} x_{i}(t)$ is invariant.

Proof. Let $\xi(t)=(1 / N) \sum_{i=1}^{N} x_{i}(t)$. For the continuous-time subsystems (5), it is proved that $\dot{\xi}(t)=(1 / N) 1_{N}^{T} L$ sat ${ }_{M}(x)=0$. Therefore, $\xi(t)$ is invariant. For the discrete-time subsystems (6), we can obtain the fact that $\xi(t+1)=(1 / N) \sum_{i=1}^{N} x_{i}(t)-$ $(1 / N) h 1_{N}^{T} L$ sat ${ }_{M}(x(t))=\xi(t)$, which implies that $\xi(t)$ is invariant. This completes the proof.

According to Lemma 2, one of the main results is given as follows. 
Theorem 3. Assume that graph $\mathscr{G}$ is connected and the condition is $0<h<1 / \max _{i \in \mathscr{I}_{N}}\left\{\sum_{j \in \mathscr{V}} a_{i j}\right\}$. Then the switched MAS with (5) and (6) can achieve consensus with arbitrary switching signals if

$$
\left|\frac{1}{N} \sum_{i=1}^{N} x_{i}(0)\right| \leq M
$$

Furthermore, the final consensus state is $\lim _{t \rightarrow \infty} x_{i}(t)=$ $(1 / N) \sum_{i=1}^{N} x_{i}(0)$.

Proof. According to Lemma 2, we have the fact that $\xi(t)=$ $(1 / N) \sum_{i=1}^{N} x_{i}(t)$ is invariant, which implies the final consensus state is $\xi=(1 / N) \sum_{i=1}^{N} x_{i}(0)$.

Consider a Lyapunov function

$$
V(t)=\sum_{i=1}^{N} x_{i}^{2}(t) \geq 0
$$

for (5) and (6). It is clear that $V(t)=0$ only when $x_{i}(t)=$ $0, i \in \mathscr{I}_{N}$. That is, $V(t)=0$ if and only if $x_{i}(t)=x_{j}(t)$.

For a time period $t_{c}$, if system (5) is switched on, we have

$$
\begin{gathered}
\dot{V}(t)=2 \sum_{i=1}^{N} x_{i}(t) \dot{x}_{i}(t)=2 \sum_{i=1}^{N} x_{i}(t) \sum_{j \in \mathscr{V}} a_{i j} x_{i}(t) \\
\cdot\left(\operatorname{sat}_{M}\left(x_{j}(t)\right)-\operatorname{sat}_{M}\left(x_{i}(t)\right)\right) \\
=-\sum_{i=1}^{N} \sum_{j \in \mathscr{V}} a_{i j}\left(x_{i}(t)-x_{j}(t)\right) \\
\cdot\left(\operatorname{sat}_{M}\left(x_{i}(t)\right)-\operatorname{sat}_{M}\left(x_{j}(t)\right)\right) \leq 0
\end{gathered}
$$

where we have used $\sum_{i=1}^{N} \sum_{j \in \mathscr{V}} a_{i j} \varepsilon_{i}\left(\zeta_{i}-\zeta_{j}\right)=$ $(1 / 2) \sum_{i=1}^{N} \sum_{j \in \mathscr{V}} a_{i j}\left(\varepsilon_{i}-\varepsilon_{j}\right)\left(\zeta_{i}-\zeta_{j}\right)$ for any $\varepsilon_{i}, \zeta_{i} \in \mathbb{R}, i=$ $1,2, \ldots, N$, under the undirected and connected graph. Since we know $\operatorname{sign}\left(\operatorname{sat}_{M}\left(x_{i}(t)\right)-\operatorname{sat}_{M}\left(x_{j}(t)\right)\right)=\operatorname{sign}\left(x_{i}(t)-x_{j}(t)\right)$, there exists some constant $\epsilon_{1}>0$ such that $1 \geq\left(\operatorname{sat}_{M}\left(x_{i}(t)\right)\right.$ $\left.\operatorname{sat}_{M}\left(x_{j}(t)\right)\right) /\left(x_{i}(t)-x_{j}(t)\right) \geq \epsilon_{1}$. Then, we have

$$
\begin{aligned}
& \dot{V}(t)=-\sum_{i=1}^{N} \sum_{j \in \mathscr{V}} a_{i j}\left(x_{i}(t)-x_{j}(t)\right) \\
& \cdot\left(\operatorname{sat}_{M}\left(x_{i}(t)\right)-\operatorname{sat}_{M}\left(x_{j}(t)\right)\right) \\
& \leq-\epsilon_{1} \sum_{i=1}^{N} \sum_{j \in \mathscr{V}} a_{i j}\left(x_{i}(t)-x_{j}(t)\right)^{2}=-\epsilon_{1} \lambda_{2} V_{1}(t),
\end{aligned}
$$

where $\lambda_{2}>0$.
For a period $t_{d}$, if the discrete-time subsystem (6) is activated, the time shift of $V(t)$ is

$$
\begin{aligned}
& \triangle V(t)=\sum_{i=1}^{N} x_{i}^{2}(t+1)-\sum_{i=1}^{N} x_{i}^{2}(t)=-\sum_{i=1}^{N}\left(2 x_{i}(t)\right. \\
& \left.-h \sum_{j \in \mathscr{V}} a_{i j}\left(\operatorname{sat}_{M}\left(x_{i}(t)\right)-\operatorname{sat}_{M}\left(x_{j}(t)\right)\right)\right) \\
& \times h \sum_{j \in \mathscr{V}} a_{i j}\left(\operatorname{sat}_{M}\left(x_{i}(t)\right)-\operatorname{sat}_{M}\left(x_{j}(t)\right)\right) \\
& =-h \sum_{i=1}^{N} \sum_{j \in \mathscr{V}} a_{i j}\left(x_{i}(t)-x_{j}(t)\right) \\
& \cdot\left(\operatorname{sat}_{M}\left(x_{i}(t)\right)-\operatorname{sat}_{M}\left(x_{j}(t)\right)\right) \\
& +h^{2} \sum_{i=1}^{N}\left(\sum_{j \in \mathscr{V}} a_{i j}\left(\operatorname{sat}_{M}\left(x_{j}(t)\right)-\operatorname{sat}_{M}\left(x_{i}(t)\right)\right)\right)^{2} .
\end{aligned}
$$

According to the Cauchy-Schwarz inequality, we have

$$
\begin{aligned}
& \triangle V(t)=-h \sum_{i=1}^{N} \sum_{j \in \mathscr{V}} a_{i j}\left(x_{i}(t)-x_{j}(t)\right) \\
& \cdot\left(\operatorname{sat}_{M}\left(x_{i}(t)\right)-\operatorname{sat}_{M}\left(x_{j}(t)\right)\right) \\
& +h^{2} \sum_{i=1}^{N}\left(\sum_{j \in \mathscr{V}} \sqrt{a_{i j}} \sqrt{a_{i j}}\left(\operatorname{sat}_{M}\left(x_{j}(t)\right)-\operatorname{sat}_{M}\left(x_{i}(t)\right)\right)\right)^{2} \\
& \leq-h \sum_{i=1}^{N} \sum_{j \in \mathscr{V}} a_{i j}\left(x_{i}(t)-x_{j}(t)\right) \\
& \cdot\left(\operatorname{sat}_{M}\left(x_{i}(t)\right)-\operatorname{sat}_{M}\left(x_{j}(t)\right)\right) \\
& +h^{2} \sum_{i=1}^{N} \sum_{j \in \mathscr{V}} a_{i j} \sum_{j \in \mathscr{V}} a_{i j}\left(\operatorname{sat}_{M}\left(x_{j}(t)\right)-\operatorname{sat}_{M}\left(x_{i}(t)\right)\right)^{2} \\
& =-h \sum_{i=1}^{N} \sum_{j \in \mathscr{V}} a_{i j}\left(\operatorname{sat}_{M}\left(x_{i}(t)\right)-\operatorname{sat}_{M}\left(x_{j}(t)\right)\right) \\
& \quad \times\left(\left(x_{i}(t)-x_{j}(t)\right)\right. \\
& \left.\quad-h \sum_{j \in \mathscr{V}} a_{i j}\left(\operatorname{sat}_{M}\left(x_{i}(t)\right)-\operatorname{sat}_{M}\left(x_{j}(t)\right)\right)\right) \leq 0,
\end{aligned}
$$

where the fact is that the sign of $\left(x_{i}(t)-x_{j}(t)\right)-$ $h \sum_{j \in \mathscr{V}} a_{i j}\left(\operatorname{sat}_{M}\left(x_{i}(t)\right)-\operatorname{sat}_{M}\left(x_{j}(t)\right)\right)$ is equivalent to the sign of $x_{i}(t)-x_{j}(t)$, since $0<h<1 / \max _{i \in \mathcal{I}_{N}}\left\{\sum_{j \in \mathscr{V}} a_{i j}\right\}$. Furthermore, we define $\left(x_{i}(t)-x_{j}(t)\right)-h \sum_{j \in \mathscr{V}} a_{i j}\left(\operatorname{sat}_{M}\left(x_{i}(t)\right)-\right.$ 
$\left.\operatorname{sat}_{M}\left(x_{j}(t)\right)\right)=\epsilon_{2}\left(x_{i}(t)-x_{j}(t)\right)$, where $0<\epsilon_{2}<1$. Then, we have

$$
\begin{aligned}
& \triangle V(t) \leq-\epsilon_{2} h \sum_{i=1}^{N} \sum_{j \in \mathscr{V}} a_{i j}\left(x_{i}(t)-x_{j}(t)\right) \\
& \cdot\left(\operatorname{sat}_{M}\left(x_{i}(t)\right)-\operatorname{sat}_{M}\left(x_{j}(t)\right)\right) \leq-\epsilon_{1} \epsilon_{2} h \lambda_{2} V(t) .
\end{aligned}
$$

If we give a time period $t=t_{c}+t_{d}$, where $t_{c}$ is the time for the continuous-time system (5) and $t_{d}$ is the time for the discretetime system (6), then we have

$$
\begin{aligned}
V(t) & \leq e^{-\epsilon_{1} \lambda_{2} t_{c}}\left(1-\epsilon_{1} \epsilon_{2} h \lambda_{2}\right)^{t_{d}} V(0) \\
& =e^{-\epsilon_{1} \lambda_{2} t_{c}} e^{-t_{d} \ln \left(1 /\left(1-\epsilon_{1} \epsilon_{2} h \lambda_{2}\right)\right)} V(0) \leq e^{-2 \gamma t} V(0),
\end{aligned}
$$

where $\gamma=\min \left\{\epsilon_{1} \lambda_{2} / 2, \ln \left(1 /\left(1-\epsilon_{1} \epsilon_{2} h \lambda_{2}\right)\right) / 2\right\}$. It follows that $\left|x_{i}(t)\right| \leq e^{-\gamma t} x_{i}(0), i \in \mathscr{I}_{N}$. Furthermore, as for $\left|(1 / N) \sum_{i=1}^{N} x_{i}(0)\right| \leq M$, we know from (11) and (14) that $\dot{V}(t)=0$ or $\Delta V(t)=0$ only if $x_{i}(t)=x_{j}(t)$ or $\operatorname{sat}_{M}\left(x_{i}(t)\right)=\operatorname{sat}_{M}\left(x_{j}(t)\right), i, j \in \mathscr{I}_{N}$. Thus, the switched MAS with (5) and (6) can achieve consensus if $\operatorname{sat}_{M}\left(x_{i}(t)\right)=$ $\operatorname{sat}_{M}\left(x_{j}(t)\right), i, j \in \mathscr{I}_{N}$, can make $x_{i}(t)=x_{j}(t), i, j \in \mathscr{I}_{N}$. Note that $\operatorname{sat}_{M}\left(x_{i}(t)\right)=\operatorname{sat}_{M}\left(x_{j}(t)\right), i, j \in \mathscr{I}_{N}$, only when $x_{i}(t) \geq M$ or $x_{i}(t) \leq-M$ and $\left|x_{i}(t)\right| \leq M$ with $x_{1}(t)=x_{2}(t)=$ $\cdots=x_{N}(t)$. Since $\left|(1 / N) \sum_{i=1}^{N} x_{i}(0)\right| \leq M$, then the condition that $x_{i}(t) \geq M$ or $x_{i}(t) \leq-M$ is not satisfied. Therefore, sat $_{M}\left(x_{i}(t)\right)=\operatorname{sat}_{M}\left(x_{j}(t)\right)$ only when $x_{i}(t)=x_{j}(t), i, j \in \mathscr{I}_{N}$ is satisfied. This completes the proof.

Remark 4. With the Gershgorin disk theorem, the maximal eigenvalues of $L$ are satisfied: $\lambda_{N} \leq 2 \max _{i \in \mathcal{I}_{N}}\left\{d_{i i}\right\}=$ $2 \max _{i \in \mathscr{I}_{N}}\left\{\sum_{j \in \mathscr{V}} a_{i j}\right\}$. Thus, the sampling period can be replaced by $0<h<2 / \lambda_{N}$.

Remark 5. Note that if $t_{c}=0$ (or $t_{d}=0$ ), the consensus of the switched MAS becomes the standard consensus of single discrete-time (or continuous-time) MAS, and it is easy to show that the obtained setup (9) is correct.

In what follows, we consider the switched MAS with (5) and (6) on switched and undirected graphs. That is, the graph $\mathscr{G}(t)$ will be randomly switched among $s$ distinct and finite topologies $\mathscr{G}(t) \in\left\{\mathscr{G}_{1}, \mathscr{G}_{2}, \ldots, \mathscr{G}_{s}\right\}$, and $\mathscr{G}(t)=\mathscr{G}_{k}$ if and only if the random switching signal $\sigma(t)=k \in\{1,2, \ldots, s\}$. Then, the following results can be obtained.

Theorem 6. Assume graph $\mathscr{G}_{k}$ is undirected and connected for each $\mathscr{G}_{k} \in\left\{\mathscr{G}_{1}, \mathscr{G}_{2}, \ldots, \mathscr{G}_{s}\right\}$ and the condition is $0<h<$ $\min _{k \in\{1,2, \ldots, s\}}\left(2 / \lambda_{N}\left(L_{k}\right)\right)$, where the Laplacian matrix of graph $\mathscr{G}_{k}$ is $L_{k}$. Then, the switched MAS with (5) and (6) can solve the consensus problem if

$$
\left|\frac{1}{N} \sum_{i=1}^{N} x_{i}(0)\right| \leq M .
$$

Furthermore, the final consensus state is $\lim _{t \rightarrow \infty} x_{i}(t)=$ $(1 / N) \sum_{i=1}^{N} x_{i}(0)$.
Proof. Similar to Theorem 3, it is proved that the average of all agents' states $(1 / N) \sum_{i=1}^{N} x_{i}(t)$ is invariant, which implies the final consensus state is $\xi=(1 / N) \sum_{i=1}^{N} x_{i}(0)$ if the consensus is reached.

In view of the proof of Theorem 3 , for a time period $t_{c}$, if system (5) is switched on, we have

$$
\begin{gathered}
\dot{V}(t)=-\sum_{i=1}^{N} \sum_{j \in \mathscr{V}} a_{i j}(t)\left(x_{i}(t)-x_{j}(t)\right) \\
\cdot\left(\operatorname{sat}_{M}\left(x_{i}(t)\right)-\operatorname{sat}_{M}\left(x_{j}(t)\right)\right) \leq-\epsilon_{1} \lambda_{2}(L(t)) \\
\cdot V(t) \leq-\epsilon_{1} \theta V(t),
\end{gathered}
$$

where $\theta=\min _{k \in\{1,2, \ldots, s\}} \lambda_{2}\left(L_{k}\right)$ and $\epsilon_{1}$ has been defined before.

For a time period $t_{d}$, if the discrete-time system (6) is switched on, the time shift of $V(t)$ is shown as

$\triangle V(t)$

$$
\begin{aligned}
& \leq-h \sum_{i=1}^{N} \sum_{j \in \mathscr{V}} a_{i j}(t)\left(\operatorname{sat}_{M}\left(x_{i}(t)\right)-\operatorname{sat}_{M}\left(x_{j}(t)\right)\right) \\
& \times\left(\left(x_{i}(t)-x_{j}(t)\right)\right. \\
& \left.-h \sum_{j \in \mathscr{V}} a_{i j}(t)\left(\operatorname{sat}_{M}\left(x_{i}(t)\right)-\operatorname{sat}_{M}\left(x_{j}(t)\right)\right)\right) \leq 0,
\end{aligned}
$$

since $0<h<\min _{k \in\{1,2, \ldots, s\}}\left(2 / \lambda_{N}\left(L_{k}\right)\right)$. It is clear that $V(t)=0$ if and only if $x_{i}(t)=x_{j}(t)$. Furthermore, we have

$$
\begin{aligned}
& \triangle V(t) \leq-h \sum_{i=1}^{N} \sum_{j \in \mathscr{V}} a_{i j}(t) \\
& \cdot\left(\operatorname{sat}_{M}\left(x_{i}(t)\right)-\operatorname{sat}_{M}\left(x_{j}(t)\right)\right) \times\left(\left(x_{i}(t)\right.\right. \\
& \left.-x_{j}(t)\right)-h \sum_{j \in \mathscr{V}} a_{i j}(t) \\
& \left.\cdot\left(\operatorname{sat}_{M}\left(x_{i}(t)\right)-\operatorname{sat}_{M}\left(x_{j}(t)\right)\right)\right) \\
& \leq-\epsilon_{2} h \sum_{i=1}^{N} \sum_{j \in \mathscr{V}} a_{i j}(t)\left(x_{i}(t)-x_{j}(t)\right) \\
& \cdot\left(\operatorname{sat}_{M}\left(x_{i}(t)\right)-\operatorname{sat}_{M}\left(x_{j}(t)\right)\right) \leq-\epsilon_{1} \epsilon_{2} h \theta V(t),
\end{aligned}
$$

where $\epsilon_{2}$ has been defined in Theorem 3. For any $t=t_{c}+t_{d}$, we have

$$
\begin{aligned}
V(t) & \leq e^{-\epsilon_{1} \theta t_{c}}\left(1-\epsilon_{1} \epsilon_{2} h \theta\right)^{t_{d}} V(0) \\
& =e^{-\epsilon_{1} \theta t_{c}} e^{-t_{d} \ln \left(1 /\left(1-\epsilon_{1} \epsilon_{2} h \theta\right)\right)} V(0) \leq e^{-2 \eta t} V(0),
\end{aligned}
$$




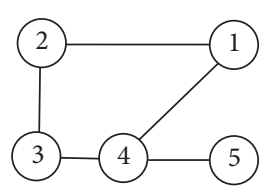

(a)

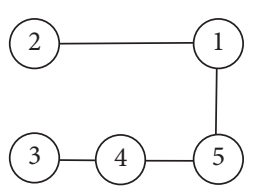

(b)

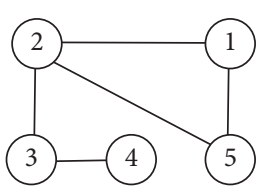

(c)

FIGURE 1: The structure of switching topology with 3 fixed and undirected graphs.
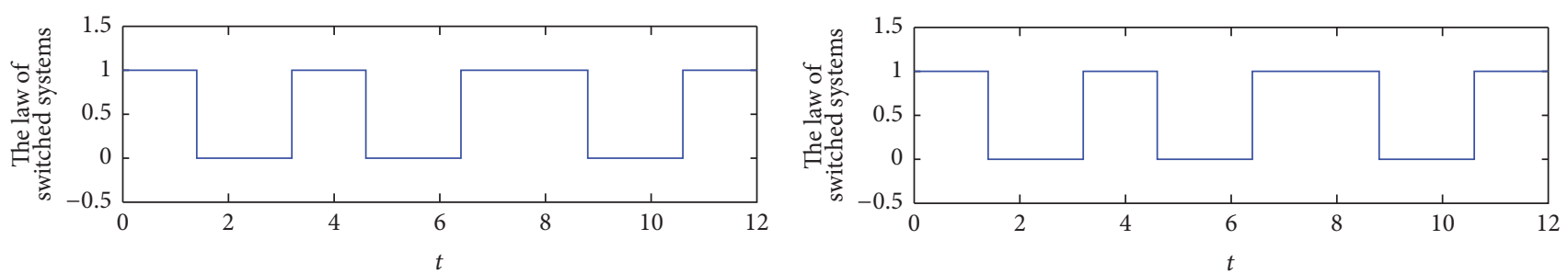

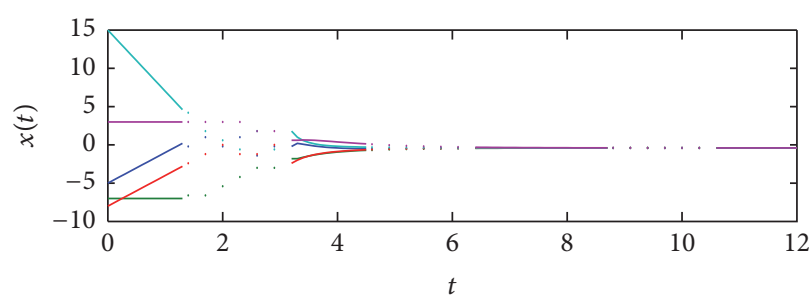

(a) $\left|(1 / 5) \sum_{i=1}^{5} x_{i}(0)\right|=0.4$

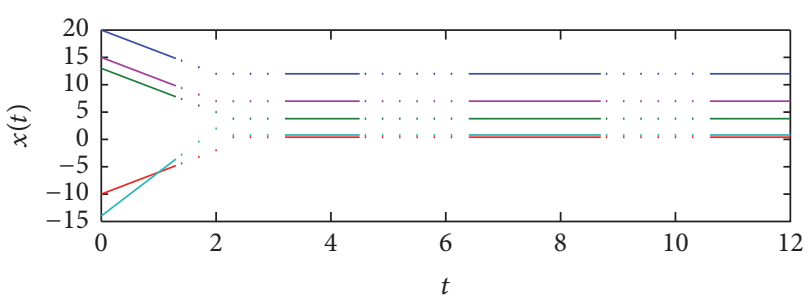

(b) $\left|(1 / 5) \sum_{i=1}^{5} x_{i}(0)\right|=4.8$

Figure 2: Consensus of switched systems on undirected graph with fixed topologies.

where $\eta=\min \left\{\epsilon_{1} \theta / 2, \ln \left(1 /\left(1-\epsilon_{1} \epsilon_{2} h \theta\right)\right) / 2\right\}$. It follows that $\left|x_{i}(t)\right| \leq e^{-\eta t} x_{i}(0), i \in \mathscr{I}_{N}$. Then, similar to the proof of Theorem 3, since $\left|(1 / N) \sum_{i=1}^{N} x_{i}(0)\right| \leq M$, we have $\dot{V}(t)=0$ or $\triangle V(t)=0$ only when $x_{i}(t)=x_{j}(t), i, j \in \mathscr{I}_{N}$. Thus, the switched MAS with (5) and (6) can achieve consensus, which completes the proof.

\section{Simulations}

This section will present two examples to illustrate the results on the fixed and switching undirected graphs.

Example 1. Let the fixed undirected graph be given as Figure 1(a). According to Theorem 3, the fact that sampling period condition is $0<h<0.4463$ can be calculated. Let $h=0.3$ and $M=2$ in this example. Figure 2 shows the law of the switched MAS and the trajectories of agents under different initial conditions. In Figure 2, the switching signals of switched systems are presented by 1 and 0 , where the continuous-time systems are presented by the signal 1 and the discrete-time systems are presented by the signal 0 . Figure $2(a)$ is with the initial states $x^{1}(0)=$ $[-5,-7,-8,15,3]^{T}$, and Figure $2(\mathrm{~b})$ is with the initial states $x^{2}(0)=[20,13,-10,-14,15]^{T}$. Under the initial states $x^{1}(0)$, the absolute final value is $\left|(1 / 5) \sum_{i=1}^{5} x_{i}(0)\right|=0.4 \leq M=2$, which satisfies condition (9), and then the switched MAS can achieve consensus under arbitrarily given switching law. However, under the initial states $x^{2}(0)$, the absolute final value is $\left|(1 / 5) \sum_{i=1}^{5} x_{i}(0)\right|=4.8 \geq M=2$ in Figure $2(\mathrm{~b})$, which does not satisfy condition (9), and thus the switched MAS cannot achieve consensus.

Example 2. Suppose that the switching undirected graph is given in Figure 1, and the switching set $\mathscr{G}(t)=\mathscr{G}_{k} \in$ $\left\{\mathscr{G}_{a}, \mathscr{G}_{b}, \mathscr{G}_{c}\right\}$. The sampling period condition can be calculated as $0<h<\min \{0.4463,0.5528,0.4796\}=0.4463$. Let $h=0.4$ and $M=5$. In the top panel of Figure 3, we give the law of switching topologies, where the signals 1,2, and 3 represent the graphs $\mathscr{G}_{a}, \mathscr{G}_{b}$, and $\mathscr{G}_{c}$, respectively. The law of switched systems is shown in the middle panel of Figure 3. In the bottom panel of Figure 3, we give the trajectories of agents with the initial states $x^{3}(0)=[2,17,-15,-10,-5]^{T}$. The figure shows that, with the condition $\left|(1 / 5) \sum_{i=1}^{5} x_{i}^{3}(0)\right|=$ $2.2<5$ in Theorem 6, the switched MAS can achieve consensus.

\section{Conclusions}

In this study, the consensus was studied for the switched MAS with relative state constraints. Since saturation constraints have been considered, the switched MAS should satisfy some conditions for achieving consensus. Therefore, considering the switched MAS with saturations, we have proposed the conditions for fixed and switching undirected graphs. Furthermore, the general magnitude saturation function has been considered. Then, by employing some standard assumptions, sufficient conditions have been provided for 

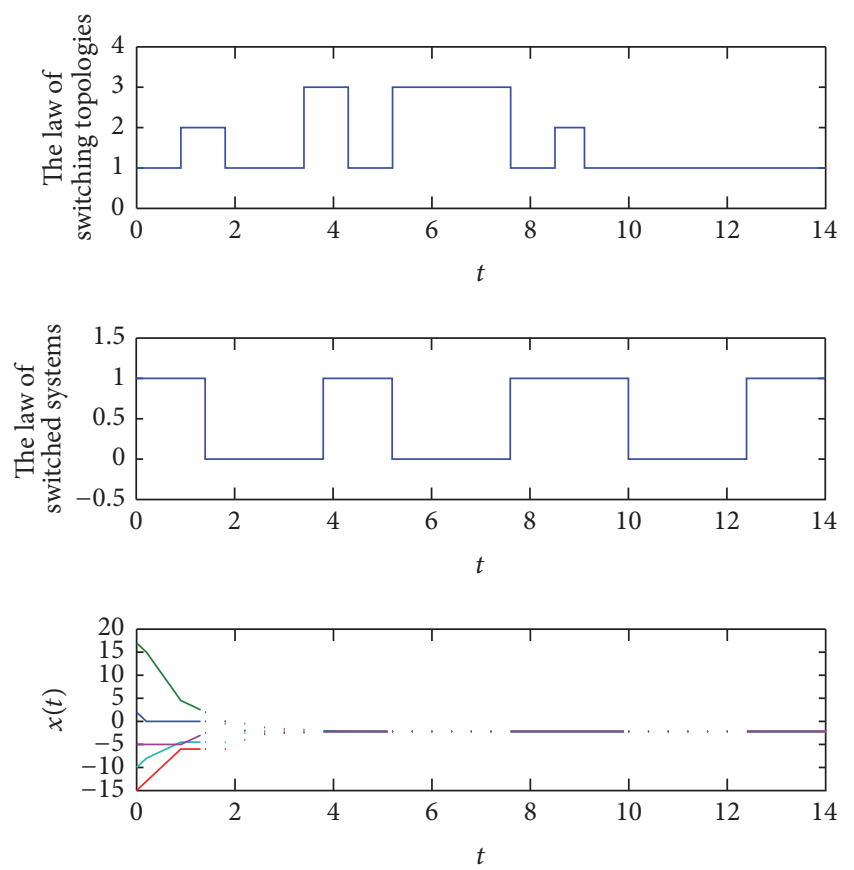

FIGURE 3: Consensus of switched systems on undirected graph with switching topologies.

the switched MAS with state constraints. Finally, we have presented two examples to verify the main results.

\section{Conflicts of Interest}

The author declares that they have no conflicts of interest.

\section{Acknowledgments}

This work was partially supported by the National Natural Science Foundation of China (61503079), the Jiangsu Natural Science Foundation (BK20150625), the Fundamental Research Funds for the Central Universities, and a project funded by the Priority Academic Program Development of Jiangsu Higher Education Institutions.

\section{References}

[1] A. Jadbabaie, J. Lin, and A. S. Morse, "Coordination of groups of mobile autonomous agents using nearest neighbor rules," Institute of Electrical and Electronics Engineers Transactions on Automatic Control, vol. 48, no. 6, pp. 988-1001, 2003.

[2] R. Olfati-Saber and R. M. Murray, "Consensus problems in networks of agents with switching topology and time-delays," Institute of Electrical and Electronics Engineers Transactions on Automatic Control, vol. 49, no. 9, pp. 1520-1533, 2004.

[3] Y. Cao, W. Yu, W. Ren, and G. Chen, "An overview of recent progress in the study of distributed multi-agent coordination," IEEE Transactions on Industrial Informatics, vol. 9, no. 1, pp. 427-438, 2013.

[4] J. Huang, H. Fang, L. Dou, and J. Chen, "An overview of distributed high-order multi-agent coordination," IEEE/CAA Journal of Automatica Sinica, vol. 1, no. 1, pp. 1-9, 2014.
[5] Z. Li, Z. Duan, G. Chen, and L. Huang, "Consensus of multiagent systems and synchronization of complex networks: a unified viewpoint," IEEE Transactions on Circuits and Systems I: Regular Papers, vol. 57, no. 1, pp. 213-224, 2010.

[6] G. Chen, C. J. Li, and Z. Y. Dong, "Parallel and distributed computation for dynamical economic dispatch," IEEE Transactions on Smart Grid, vol. 8, no. 2, pp. 1026-1027, 2017.

[7] C. Li, X. Yu, W. Yu, G. Chen, and J. Wang, "Efficient computation for sparse load shifting in demand side management," IEEE Transactions on Smart Grid, vol. 8, no. 1, pp. 250-261, 2016.

[8] S. Liu, L. Xie, and F. L. Lewis, "Synchronization of multiagent systems with delayed control input information from neighbors," Automatica, vol. 47, no. 10, pp. 2152-2164, 2011.

[9] W. Yu, G. Chen, M. Cao, J. Lü, and H.-T. Zhang, "Swarming behaviors in multi-agent systems with nonlinear dynamics," Chaos: An Interdisciplinary Journal of Nonlinear Science, vol. 23, no. 4, Article ID 043118, 2013.

[10] W. Li, H.-T. Zhang, M. Z. Chen, and T. Zhou, "Singularities and symmetry breaking in swarms," Physical Review E: Statistical, Nonlinear, and Soft Matter Physics, vol. 77, no. 2, Article ID 021920, 2008.

[11] Y. Zheng, Y. Zhu, and L. Wang, "Consensus of heterogeneous multi-agent systems," IET Control Theory \& Applications, vol. 5, no. 16, pp. 1881-1888, 2011.

[12] Y. Hong, J. Hu, and L. Gao, "Tracking control for multiagent consensus with an active leader and variable topology," Automatica, vol. 42, no. 7, pp. 1177-1182, 2006.

[13] U. Munz, A. Papachristodoulou, and F. Allgower, "Consensus in multi-agent systems with coupling delays and switching topology," Institute of Electrical and Electronics Engineers Transactions on Automatic Control, vol. 56, no. 12, pp. 2976-2982, 2011.

[14] G. S. Seyboth, D. V. Dimarogonas, and K. H. Johansson, "Event-based broadcasting for multi-agent average consensus," Automatica, vol. 49, no. 1, pp. 245-252, 2013.

[15] Y. Zheng and L. Wang, "Finite-time consensus of heterogeneous multi-agent systems with and without velocity measurements," Systems \& Control Letters, vol. 61, no. 8, pp. 871-878, 2012.

[16] G. Xie and L. Wang, "Consensus control for a class of networks of dynamic agents," International Journal of Robust and Nonlinear Control, vol. 17, no. 10-11, pp. 941-959, 2007.

[17] W. Ren, "On consensus algorithms for double-integrator dynamics," Institute of Electrical and Electronics Engineers. Transactions on Automatic Control, vol. 53, no. 6, pp. 1503-1509, 2008.

[18] W. Ni and D. Cheng, "Leader-following consensus of multiagent systems under fixed and switching topologies," Systems and Control Letters, vol. 59, no. 3-4, pp. 209-217, 2010.

[19] S. Liu, L. Xie, and H. Zhang, "Distributed consensus for multiagent systems with delays and noises in transmission channels," Automatica, vol. 47, no. 5, pp. 920-934, 2011.

[20] W. W. Yu, G. R. Chen, M. Cao, and J. Kurths, "Second-order consensus for multiagent systems with directed topologies and nonlinear dynamics," IEEE Transactions on Systems, Man, and Cybernetics, Part B: Cybernetics, vol. 40, no. 3, pp. 881-891, 2010.

[21] K. You and L. Xie, "Network topology and communication data rate for consensusability of discrete-time multi-agent systems," Institute of Electrical and Electronics Engineers Transactions on Automatic Control, vol. 56, no. 10, pp. 2262-2275, 2011.

[22] G. Wen, Z. Duan, W. Yu, and G. Chen, "Consensus in multiagent systems with communication constraints," International Journal of Robust and Nonlinear Control, vol. 22, no. 2, pp. 170$182,2012$. 
[23] Y. Li, J. Xiang, and W. Wei, "Consensus problems for linear timeinvariant multi-agent systems with saturation constraints," IET Control Theory \& Applications, vol. 5, no. 6, pp. 823-829, 2011.

[24] A. Abdessameud and A. Tayebi, "On consensus algorithms design for double integrator dynamics," Automatica, vol. 49, no. 1, pp. 253-260, 2013.

[25] Q. Wang, C. Yu, and H. Gao, "Semiglobal synchronization of multiple generic linear agents with input saturation," International Journal of Robust and Nonlinear Control, vol. 24, no. 18, pp. 3239-3254, 2014.

[26] T. Yang, Z. Meng, D. V. Dimarogonas, and K. H. Johansson, "Global consensus for discrete-time multi-agent systems with input saturation constraints," Automatica, vol. 50, no. 2, pp. 499-506, 2014.

[27] H. Su, M. Z. Chen, J. Lam, and Z. Lin, "Semi-global leaderfollowing consensus of linear multi-agent systems with input saturation via low gain feedback," IEEE Transactions on Circuits and Systems I: Regular Papers, vol. 60, no. 7, pp. 1881-1889, 2013.

[28] Z.-X. Liu and Z.-Q. Chen, "Discarded consensus of network of agents with state constraint," Institute of Electrical and Electronics Engineers. Transactions on Automatic Control, vol. 57, no. 11, pp. 2869-2874, 2012.

[29] Y.-H. Lim and H.-S. Ahn, "Consensus under saturation constraints in interconnection states," Institute of Electrical and Electronics Engineers Transactions on Automatic Control, vol. 60, no. 11, pp. 3053-3058, 2015.

[30] Q. Wang and C. Sun, "Conditions for consensus in directed networks of agents with heterogeneous output saturation," IET Control Theory \& Applications, vol. 10, no. 16, pp. 2119-2127, 2016.

[31] G. Zhai, D. Liu, J. Imae, and T. Kobayashi, "Lie algebraic stability analysis for switched systems with continuous-time and discrete-time subsystems," IEEE Transactions on Circuits and Systems II: Express Briefs, vol. 53, no. 2, pp. 152-156, 2006.

[32] Y. Zheng and L. Wang, "Consensus of switched multiagent systems," IEEE Transactions on Circuits and Systems II: Express Briefs, vol. 63, no. 3, pp. 314-318, 2016.

[33] X. Lin, Y. Zheng, and L. Wang, "Consensus of switched multiagent systems with random networks," International Journal of Control, vol. 90, no. 5, pp. 1113-1122, 2017.

[34] X. Lin and Y. Zheng, "Finite-time consensus of switched multiagent systems," IEEE Transactions on Systems, Man, and Cybernetics: Systems, vol. 47, no. 7, pp. 1535-1545, 2016.

[35] Y. Zhu, Y. Zheng, and L. Wang, "Containment control of switched multi-agent systems," International Journal of Control, vol. 88, no. 12, pp. 2570-2577, 2015. 


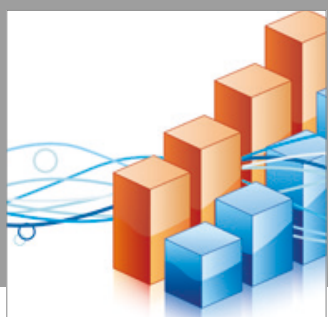

Advances in

Operations Research

vatersals

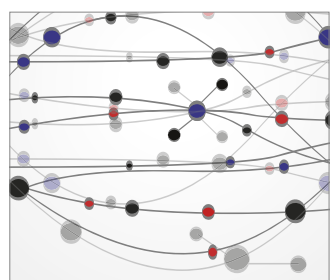

\section{The Scientific} World Journal
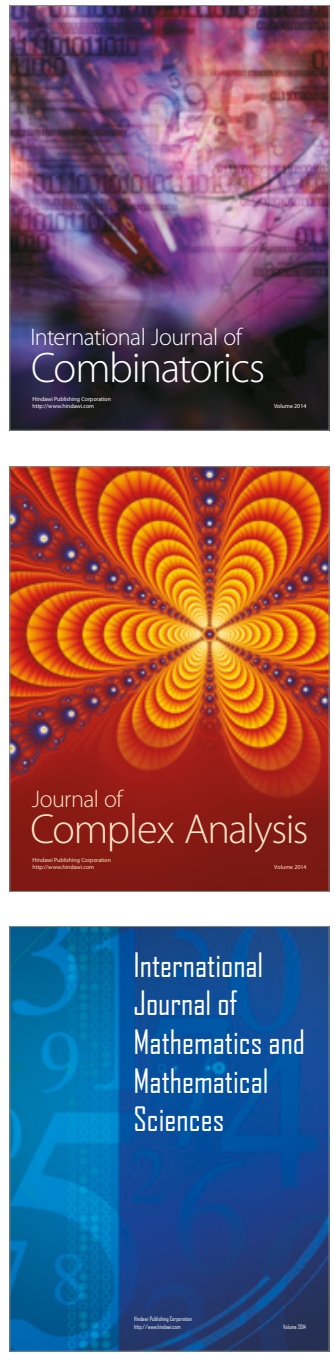
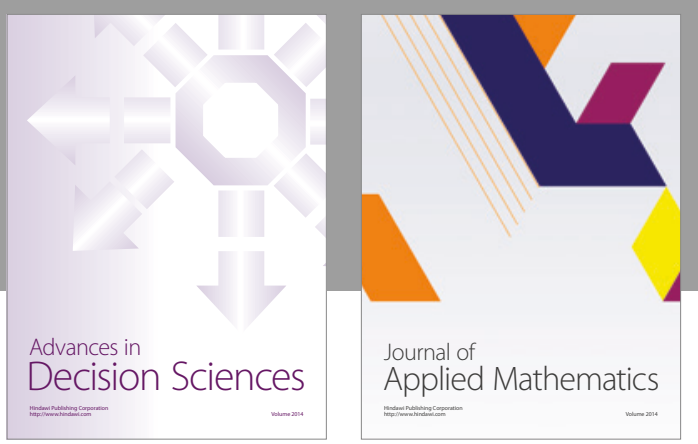

Algebra

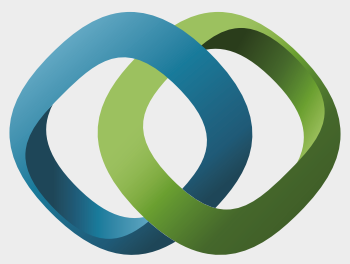

\section{Hindawi}

Submit your manuscripts at

https://www.hindawi.com
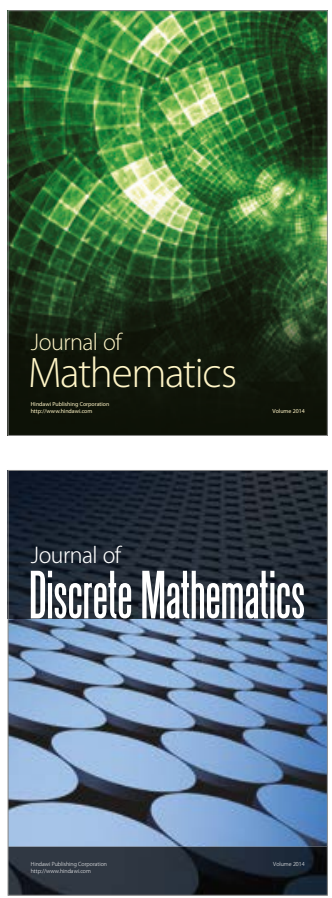

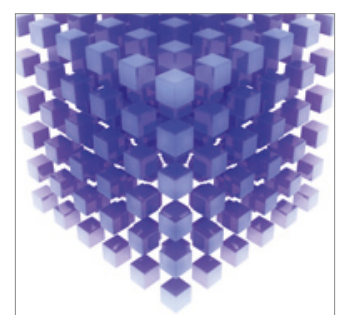

Mathematical Problems in Engineering
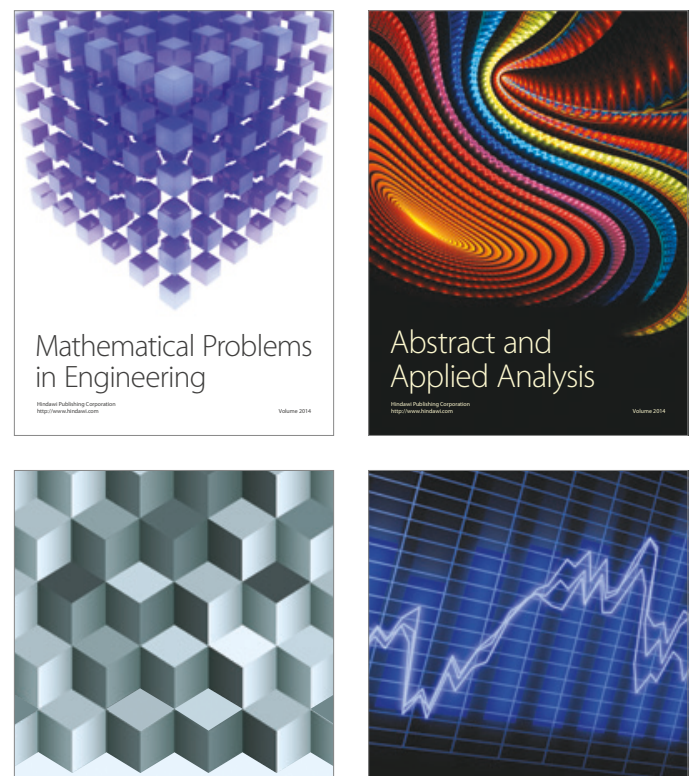

Journal of

Function Spaces

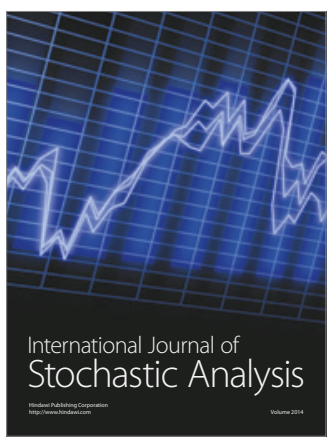

Probability and Statistics
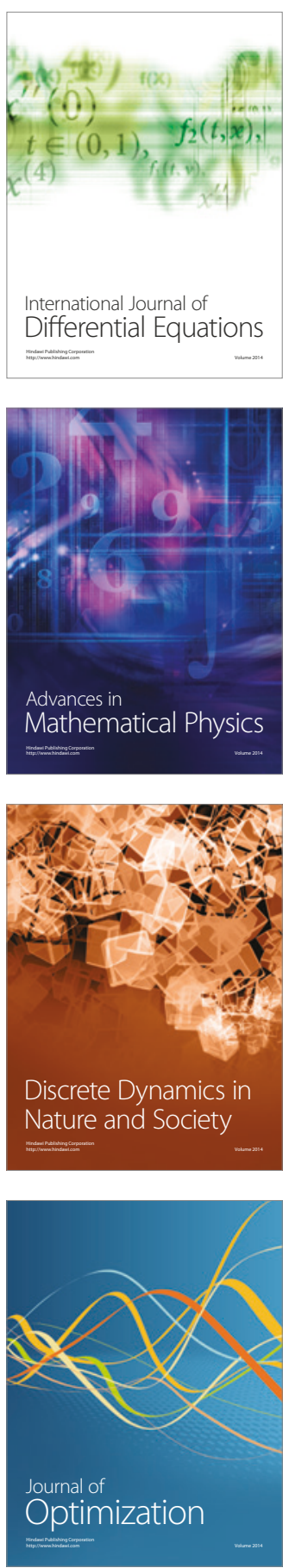